Article

\title{
Ang Pilosopiya ni Jean Baudrillard bilang Batayang Teoretikal sa Araling Pilipino
}

\section{F.P.A. Demeterio III and Emmanuel C. de Leon}

\begin{abstract}
This paper is basically a presentation of the tenets of Jean Baudrillard's philosophy in a language and level that can be easily understood by Filipino students and scholars of philosophy, cultural studies and Philippine studies. The discussion of Baudrillard's philosophy revolves around 1) his Marxist phase, 2) his anti-Marxist phase, and 3) postmodern phase. The ultimate aim of this paper is to suggest some aspects and dimensions of Philippine society and culture that can be analyzed using some of Baudrillard's thoughts as interpretive frameworks, as well as to challenge the said Filipino students and scholars of philosophy, cultural studies and Philippine studies to creatively and effectively appropriate such theories for the enrichment of the theoretical corpus of Philippine studies. This paper is part of a series of similar works done by one of the co-authors that dealt with Adorno, Schleiermacher and Dilthey, Heidegger, Gadamer and Bultmann, Lyotard, and Bourdieu.
\end{abstract}

Keywords: Buadrillard, Philippine studies, postmodernism, hyperreality

\section{Introduksiyon}

$\mathrm{H}$

angarin ng papel na itong mailahad ang mga mahalagang aspekto ng kaisipan ni Jean Baurillard (1929-2007), Pranses na sosyolihista, pangkulutral na kritiko, pilosopo, at isa sa mga pangunahing teorisista ng posmodernismo. Hindi lamang inog ang papel sa wikang Filipino, bagkus ipapamalas ang antas ng diskursong maiintindihan ng mga kapwa Pilipinong nasa larangan ng araling kultural at araling Pilipino. Makatutulong ito sa mga wala pa masyadong kasanayan sa antas ng diskursong pilosopikal, sa mga Pilipinong mag-aaral ng pilosopiyang marahil hindi pa handang basahin ang mga primaryong teksto ni Baudrillard o ang mga komplikadong babasahin tungkol sa kanya na naisulat na sa wikang Ingles.

(c) 2015 F.P.A. Demeterio III and Emmanuel C. de Leon http://www.kritike.org/journal/issue 16/demeterio\&de leon june2015.pdf ISSN 1908-7330 
Tunguhin din ng papel na itong matukoy ang ilang aspekto mula sa naturang banyagang diskurso para lalong mapagyaman ang teoretikal na korpus ng lokal na araling kultural at araling Pilipino.

Ipinanganak si Baudrillard noong 1929 sa Reims, isang tanyag na lunsod sa Pransia dahil sa kanyang nakamamanghang Gotikong katedral. Ayon kay Baudrillard, ang kanilang angkan ay mga magsasaka, subalit ang kanyang mga magulang ay naging mga kawani naman ng gobiyerno. ${ }^{1}$ Doon sa Sorbonne University sa Paris, pinag-aralan ni Baudrillard ang Aleman. Sa mga panahong iyon labis siyang nababalisa tungkol sa sumisiklab na digmaan sa Algeria, na noon ay isang kolonya ng Pransia. ${ }^{2}$

Bilang tapos sa araling Aleman, nagturo si Baudrillard ng kultura at wikang ito sa isang sekondaryang paaralan, habang nagsasalin sa wikang Pranses ng mga aklat ng Aleman-Suizong manunulat at pintor na si Peter Weiss (1916-1982), ng Alemang Marxistang manunulat at direktor ng teatrong si Bertolt Brecht (1898-1956), at ng Alemang sosyolohista at antropolohistang si Wilhelm Muhlmann (1904-1988), at habang pinagaaralan niya ang mga akda ng Pranses na Marxistang sosyolohista at pilosopong si Henri Lefebvre (1901-1991), at ng Pranses na kritikong pangkultural at semyolohistang si Roland Barthes (1915-1980).

Noong 1966, pumasok siya sa University of Paris sa Nanterre; nagtrabaho bilang assistant ni Lefebvre; nag-aral ng mga wika, pilosopiya, sosyolohiya at iba pang mga kaakibat na disiplina; nagtapos ng kanyang disertasyon sa sosyolohiya, na may pamagat na Le Systeme des Objects (The System of Objects); at nagturo ng sosyolohiya. Ang Nanterre ay kilalang balwarte ng mga makakaliwang mag-aaral at guro, kaya di maikakaila ang pagiging makakaliwa ni Baudrillard at ang kanyang pagkasangkot sa pagalsa ng mga mag-aaral noong Mayo ng $1968 .^{3}$

Natapos niya ang kanyang habilitasyon, o ang pangalawang disertasyon ng mga Europeyo, noong 1972, at nakakuha ng ranggong propesor ng sosyolohiya. Noong 1987, iniwan niya ang Nanterre at tumahak sa landas ng isang independiyenteng intelektwal at interdisiplinaryong kritiko, habang tumatanggap ng pa-ilan-ilang trabaho bilang mananaliksik sa University of Paris-IX Dauphine, at bilang propesor sa European Graduate School sa Suiza. Si Baudrillard ay namatay matapos ang mahabang pakikipaglaban sa sakit na kanser noong 2007, sa edad na 77.

Masasabing ang kabuohan ng teoryang kultural ni Baudrillard ay nagkaroon ng maraming pagbabago at pag-iibang anyo mula noong dekada sisenta hangang sa unang dekada ng kasalukuyang siglo. Maaari nating

${ }^{1}$ See Mike Gane ed., Baudrillard Live: Selected Interviews (London: Routledge, 1993),

19.

2 Ibid., 20.

${ }^{3}$ Ibid., 74 .

(C) 2015 F.P.A. Demeterio III and Emmanuel C. de Leon http://www.kritike.org/journal/issue 16/demeterio\&de leon june2015.pdf

ISSN 1908-7330 
patawan ng tatlong yugto ang kanyang teoryang kultural upang mas madaling pag-aralan at unawain ito: 1) ang kanyang Marxistang yugto, mula 1968 hangang 1971; 2) ang kanyang anti-Marxistang yugto, mula 1972 hanggang 1976; at 3) ang kanyang postmodernistang yugto, mula 1976 hanggang 2007.

\section{Marxistang Yugto}

Ang Marxistang yugto sa kaisipan ni Baudrillard ay nag-umpisa sa kanyang disertasyon, na isinulat sa gabay nina Barthes at Lefebvre, at substansyal na matatagpuan sa kanyang mga aklat na The System of Objects ng 1968, The Consumer Society ng 1970, at For a Critique of the Political Economy of the Sign ng 1972. Ang pagiging Marxista ni Baudrillard sa panahong ito ay makikita sa kanyang pagbibigay ng Marxistang interpretasyon sa isang bagay na hindi masyadong pinaglaanan ng pansin ni Karl Marx (1818-1883): ang pagkukunsumo. Kung si Marx ay nakatutok sa produksyon, si Baudrillard naman ay nakatutok sa kabilang dulo ng mahabang tanikala ng modernong ekonomiya na walang iba kung hindi ang pagkukunsumo ng mga produkto.

Bago pa man ang lahat, dapat nating isa-isip na ang kapitalismong pinag-aralan ni Marx ay hindi na ang kaparehong kapitalismong pinaglalaanan ng pansin ni Baudrillard. Ang tawag ni Baudrillard sa sinaunang anyo ng kapitalismo ay "kapitalismo ng kompetitibong merkado," at sa kasalukuyang anyo naman ay "monopolyong kapitalismo." Kung ang kapitalismo ng kompetitibong merkado ay nakatutuon sa pagpapalaki ang produksyon upang maipababa ang presyo ng produkto, ang monopolyong kapitalismo, ayon kay Baudrillard, ay nakatutok sa pagpapatindi at pagpapalawak ng demand, sa pamamagitan ng patalastas, pagbalot at presentasyon, at pagmanipula ng moda. ${ }^{4}$

Ayon kay Baudrillard, noong mga taong 1920 hanggang 1960, dahil sa makabagong teknolohiya, lumawak ang produksyon na nagresulta sa pagbagsak ng mga presyo ng produkto. Ito na sana ang hinahangad ng kapitalismo ng kompetitibong merkado, na taliwas naman sa hangarin ng monopolyong kapitalismo. Nang lumaganap ang mga murang produkto, napag-isipan ng ilang kapitalista na gumawa ng mga produktong mamahalin at prestihiyoso, na taliwas naman sa hangarin ng kapitalismo ng kompetitibong merkado. ${ }^{5}$

Sa konteksto ng kapitalismo ng kompetitibong merkado, ang mga mamahalin at prestihiyosong produkto ay kadalasan mga gawa sa kamay

${ }^{4}$ See Douglas Kellner, "Jean Baudrillard", in The Standford Encyclopedia of Philosophy, (2014) <http://plato.stanford.edu/archives/win2014/entries/baudrillard/>, 30 March 2015.

${ }^{5}$ Ibid.

(C) 2015 F.P.A. Demeterio III and Emmanuel C. de Leon http://www.kritike.org/journal/issue 16/demeterio\&de leon june2015.pdf ISSN 1908-7330

(cc) BY-NC-ND 
ng mga ekspertong manlilikha at ibinebenta bilang mga obra maestro, o hindi kaya mga produktong nanggagaling sa malalayong lugar at ibinebenta bilang mga eksotikong bagay. Ngunit ang ipinapalaganap na mamahalin at prestihiyosong produkto ng monopolyong kapitalismo ay hindi mga obra maestro o mga eksotikong bagay. Marahil may kaunting angat ang kanilang materyales at pagka-yari kung ihahambing sa mga karaniwang produkto, subalit nagmumula pa rin sa pabrika at bultobultong iniluwal ang mga mamahalin at prestihiyosong produktong ito. Ang kanilang pagiging mamahalin at prestihiyoso ay naka-ugat sa kanilang pagtatanghal ng mga tusong kapitalista bilang mga mamahalin at prestihiyosong produkto. Samakatuwid, artipisyal lamang ang kanilang pagiging mamahalin at prestihiyoso.

Sa harap ng bumabahang mamahalin at prestihiyosong produkto, naisip ni Baudrillard ang pagkukulang ni Marx bilang teorisista sa konteksto ng bagong anyo ng kapitalismo. Naintindihan ni Baudrillard kung paano nakatutok lamang si Marx sa use-value at exchange-value ng mga produkto. Kapag igigiit ni Marx ang dalawang kategoryang ito, magmumukhang baliw ang mga taong bumibili ng mamahalin at prestihiyosong produkto sa halip ng mga mumurahing alternatibo. Batay sa ideya ng Amerikanong ekonomista at sosyolohistang si Thorstein Veblen (1857-1929) tungkol sa conspicuous consumption, naisip ni Baudrillard na maliban sa usapin ng use-value at exchange-value ang mga produkto ay maaari ding maging midyum ng kapangyarihan, karangyaan at karangalan. ${ }^{6}$

Sa puntong ito ipinasok ni Baudrillard ang teorya ng tanda na binuo ng Suizong semyolohistang si Ferdinand de Saussure (1857-1913) at ginamit na ni Barthes sa kanyang sariling pag-aaral ng mga tiyak na phenomenon tulad ng moda, pagkain, larawan, at iba pa. Dinagdagan ni Baudrillard ang use-value at exchange-value ni Marx sa sign-value. Ginawa niya ang komoditi bilang isang signifier na ang kaakibat na signified ay kapangyarihan, karangyaan at karangalan. Sa ganitong usapan, ang mga produkto ay binibili hindi lamang dahil sa kanilang praktikal na gamit, kung hindi dahil rin sa kanilang pagiging tanda ng panlipunang katayuan ng sinumang bumili sa kanila. Ipinaliwanag niya: "Ang pagkunsumo ay ang virtual na suma total ng lahat ng bagay at mensaheng kasalukuyang bumubuo sa mahigit kumulang isang diskurso. Ang pagkunsumo, habang ito ay makahulugan, ay isang sistematikong aksyon sa pagmanipula ng mga tanda."7 Kapag ang nabiling produkto ng isang tao ay mamahalin, magsisilbi itong tanda ng kanyang mataas na katayuan sa kanyang lipunan.

\footnotetext{
${ }^{6}$ Ibid.

${ }^{7}$ Jean Baudrillard, "The System of Objects", in Jean Baudrillard: Selected Writings, ed. by Mark Poster (Stanford, California: Stanford University Press, 1988), 22.
} 


\section{ANG PILOSOPIYA NI JEAN BAUDRILLARD}

Kung use-value lamang ang umiiral, lahat sana ng tao ay bibili lamang ng mga mumurahing produkto. Subalit, ayon sa nakikita natin, masasabi nating nagtagumpay ang mga kapitalista sa paghimok sa mga mamimili na naisin ang mga mamahalin at prestihiyosong produkto. Kaya iginiit ni Baudrillard na ang tao sa panahon ng monopolyong kapitalismo ay hindi lamang isang tagakunsumo ng use-value ng isang produkto, siya rin ay isang tagakunsumo ng sign-value.

Naniniwala si Baudrillard na ang lipunan sa ilalim ng monopolyong kapitalismo ay isinaayos sa pamamagitan ng pagkukunsumo at pagtatanghal ng mga produkto para mailathala ang identidad at katayuan ng bawat isa. Mayroon nang sariling hirarkiya ang mga produkto at sa pamamagitan ng pagkunsumo sa kanila nagkakaroon din ng hirarkiya ang bawat isa sa loob ng lipunan. Isinulat niya: "Sa antas ng indibidwal, kasama ang kanyang mga pangangailangan, kontradiksyon at negatibidad, ang sistema ay walang hugis (fluid) at diskonektado. Sa antas ng mga produkto, kasama ang lahat ng kanilang positibidad, ang sistema ay kodipikado, na uri-uri na, discontinuous, at masasabing integrado. Hindi ito interaksyon kung hindi sapilitang integrasyon ng sistema ng pangangailangan sa sistema ng mga produkto." 8

Ang ideya ni Baudrillard na ang mga tao sa kasalukuyan ay naging mga tagakunsumo ng tanda ay isang mabisang paliwanag sa lumalaganap na konsumerismo. Kung bakit ang tao sa kasalukuyan ay tila naging isang hindi mapuno-puno at hindi mabusog-busog na tagakunsumo ay dahil siya ay naging isa nang tagakonsumo ng mga immateryal na tanda sa halip na maging isang tagakunsumo lamang ng mga materyal na use-value ng mga produkto. Binigyang diin niya: "Ang kumpulsyong ito na kumunsumo ay hindi epekto ng ilang sikolohikal na sanhi ... o ng kapangyarihan ng paggagaya lamang. Kung ang pagkunsumo ay nagmistulang hindi na mapipigilan, ito ay dahil ang pagkunsumo ay isang buong ideyalistikong gawain na wala nang kinalaman ... sa pagtugon ng mga pangangailangan, $\mathrm{o}$ sa prinsipyo ng reyalidad; ito ay nabigyan na ng enerhiya sa proyektong palaging hindi napupuno." 9

May malaking pagkakaiba ang pagkonsumo ng use-value ng isang produkto sa pagkonsumo ng sign-value ng parehong produkto. Ang una ay nakakapuno at nakakabusog, samantalang ang pangalawa ay hindi. Halimbawa, ang taong bumili ng relos para gamitin bilang orasan ay hindi mangangailangan ng panibagong relos habang gumagana pa ang kanyang nabiling relos, dahil ang kanyang pangangailangan ng orasan ay napuno na o nabusog na. Subalit ang ibang tao na bumili ng relos bilang isang tanda ng

\footnotetext{
8 Ibid., 14
}

${ }^{9}$ Ibid., 25.

(C) 2015 F.P.A. Demeterio III and Emmanuel C. de Leon http://www.kritike.org/journal/issue 16/demeterio\&de leon june2015.pdf ISSN 1908-7330

(c) BY-NC-ND 
kanyang mataas na katayuan ay maaaring mangangailangan kaagad ng mas mamahalin pang relos sa oras na malaman niya na may mga kasamahan na siya na mas mamahalin pa ang suot na relos. Kaya ang pagkunsumo ng ibang taong ito sa sign-value ng relos ay hindi mapuno-puno at hindi mabusog-busog.

Sa harap ng paglaganap ng konsumerismo, sa harap ng pagkaingganyo ng tao sa mapang-akit na sign-value ng mga produkto, sa harap na pagkabaon natin sa sistema ng monopolyang kapitalismo, walang malinaw na programa o estratihiya si Baudrillard kung paano dapat lalaban ang tao. Hindi siya nagpahayag ng eksistential na pagtakwil sa sistema, at hindi rin siya nanawagan para sa isang rebolusyonaryong pagkilos. Ang sagot ni Baudrillard kung ano dapat ang gagawin ng tao sa konsumerismo at monopolyong kapitalismo ay mabubuo lamang matapos ang kanyang Marxistang yugto.

\section{Anti-Marxistang Yugto}

Kung sina Marx, Veblen at Saussure ang mga pangunahing impluwensiya ni Baudrillard sa kanyang Marxistang yugto, ang Pranses na sosyolohista at antropolohistang si Marcel Mauss (1872-1950) at Pranses na transgresibong manunulat na si Georges Bataille (1897-1962) naman ang gumabay sa kanya sa kanyang anti-Marxistang yugto na substansyal na matatagpuan sa kanyang mga aklat na The Mirror of Production ng 1973 at Symbolic Exchange and Death ng 1976. Pinuna ni Baudrillard sa yugtong ito ang pagkukulang ni Marx sa radikalismo at pagkamakakaliwa. Naniwala si Baudrillard na hindi totoo na labag si Marx sa kapitalismo at sa paghariharian ng mga burgis dahil hindi raw tumagos sa pinakaugat ng mga bagay na ito ang klasikal na kritisismo ni Marx.

Hindi rin nasiyan si Baudrillard sa suhestyon ni Marx na ang proletarya dapat ang magkontrola sa produksyon, dahil para sa kanya ang Marxistang obsesyon sa produksyon ay walang pagkakaiba sa kapitalistang obsesyon sa produksyon. ${ }^{10}$ Ipinaliwanag ni Baudrillard na kung matapos man ang inaasahang madugong rebolusyon, wala nang tunay na antikapitalistang alternatibong kaayusan si Marx bukod sa pag-asta ng proletarya bilang mga bagong kapitalista. Inihayag ni Baudrillard: "wala siyang (Marx) binagong pangunahing bagay: lalo na sa usapin tungkol sa paglikha ng tao sa kanyang sarili sa kanyang walang katupusang determinasyon, sa sa kanyang tuloy-tuloy na paglampas sa kanyang sarili patungo sa kanyang katapusan."11 Kaya, taliwas sa inaakala ng nakararami

${ }^{10}$ See Jean Baudrillard, The Mirror of Production (St. Louis, Missouri: Telos Press, 1975), 17-20.

${ }^{11} \mathrm{Ibid} ., 33$.

(C) 2015 F.P.A. Demeterio III and Emmanuel C. de Leon http://www.kritike.org/journal/issue 16/demeterio\&de leon june2015.pdf

ISSN 1908-7330 


\section{ANG PILOSOPIYA NI JEAN BAUDRILLARD}

na si Marx na ang pinakamakakaliwa sa lahat ng mga makakaliwa at pinakaradikal sa lahat ng mga radikal, naniwala si Baudrillard na konserbatibo ang tunay na diwa ni Marx.

Para kay Baudrillard ang nakakubling konserbatismo ni Marx ang tunay na dahilan kung bakit hindi sumuporta ang Partido Kumunistang Pranses sa pag-alsa ng mga mag-aaral noong 1968. ${ }^{12}$ Ang kabiguan ni Marx sa lubusang pagbatikos sa kapitalismo at sa naghari-hariang mga burgis, ang kabiguan ni Marx sa pagbigay ng reyalistikong alternatibong kaayusan na papalit sa kapitalismo, at ang kanyang mapagkunwaring radikalismo at pagkamakakaliwa ang ginawang batayan ni Baudrillard para idiin ang Marxismo bilang sukdulang lehitimasyon ng kapitalismo, ng mga burgis, at ng kunsumeristang landas ng buhay.

Matapos niyang itakwil ang Marxismo, bumuo si Baudrillard ng alternatibong programa na lalaban sa lalong lumalaganap at lumalakas na kapitalismo at kunsumerismo. Hinangad niya na ang alternatibong programang ito ay maging tunay na radikal at tunay na makakaliwa. Sa puntong ito ipinasok ni Baudrillard ang pananaliksik ni Mauss tungkol sa diskurso ng potlatch na matatagpuan sa Polynesia, Melanesia at Hilagang Kanlurang Amerika. Ang potlatch para sa mga katutubong lipunan ng mga nasabing lugar ay isang pampublikong pag-aalay ng isang tao ng mga regalo. ${ }^{13}$ Sa pananaw ng isang modernong indibidwal, lalo ng kanluraning indibidwal, walang kabuluhan at mahirap intindihin ang aksaya ng yaman na kaakibat sa isang potlatch. Ngunit sa pananaw ng mga tao sa nasabing mga katutubong lipunan, ang potlatch ay isang ritwalistikong paglalathala ng kayamanan at kapangyarihan ng sinumang nag-aalay nito. ${ }^{14} \mathrm{Sa}$ katunayan, hindi mahirap intindihin ng mga Pilipino ang diskurso ng potlatch dahil hindi ito nalalayo sa ating diskurso ng pagpipiyesta, kung saan ang may pinakamalaki, pinakamasarap, at pinakamagarbong handaan sa isang nayon ay siyang kinikilalang pinakamayaman at pinakamakapangyarihan sa nayong iyon.

Ipinaliwanag ni Mauss na ang potlatch, taliwas sa inaakala ng mga modernong kanluranin, ay isa palang makapangyarihang mekanismo para buuin at kumpunihin ang katutubong lipunan. Sa pamamagitan nito nakikilala ng bawat katutubo ang kani-kanilang katayuan sa loob ng kanilang katutubong lipunan. Hinango ni Baudrillard ang diskurso ng potlatch bilang isang sistema ng pagpapahalaga na salungat sa sistema ng pagpapahalaga na ipinapairal ng kapitalismo. Kung sa pamamagitan ng potlatch inilathala ng isang katutubong indibidwal ang kanyang panlipunang katayuan at kapangyarihan sa pamamagitan ng pagbigay,

\footnotetext{
12 See Kellner, "Jean Baudrillard."

${ }^{13}$ See Richard Lane, Jean Baudrillard (London: Routledge, 2000), 48-53.

${ }^{14}$ Ibid., 80.
}

(C) 2015 F.P.A. Demeterio III and Emmanuel C. de Leon http://www.kritike.org/journal/issue 16/demeterio\&de leon june2015.pdf ISSN 1908-7330 
inilathala naman ng isang modernong indibidwal ang kanyang panlipunang katayuan at kapangyarihan sa pamamagitan ng pag-angkin.

Pinayaman ni Baurdrillard ang diskurso ng potlatch gamit ang kaisipan ni Bataille tungkol sa heneral na ekonomiya na umiiral daw noong sinaunang panahon. ${ }^{15}$ Ayon kay Bataille, sa loob ng heneral na ekonomiya ay nagaganap ang paglulustay, pag-aaksaya, pagpipiyesta at pag-aalay. Malinaw na taliwas ang mga ito sa pagpapahalaga na umiiral sa loob ng kapitalismo kung saan pinupuri ang pagtitipid, pagtitimpi at pagsisikap sa paggawa ng produkto. Ngunit, ayon kay Bataille, hindi lamang mas nauna ang sistema ng pagpapahalaga ng heneral na ekonomiya, ito rin ay mas sang-ayon sa kalikasan na tao na talaga namang nasisiyahan sa paglulustay, pag-aaksaya, pagpipiyesta at pag-aalay. ${ }^{16}$ Kagaya sa nabanggit ni Mauss, ang ganitong diskurso ay nagbibigay ng makapangyarihang damdamin sa taong naglulustay, nag-aaksaya, nagpipiyesta o nag-aalay ng potlatch.

Gamit ang kaisipan nina Mauss at Bataille, binuo ni Baudrillard ang konsepto ng simbolikong pagpapalitan kung saan iminungkahi niya na ang tunay na paraan para mapuksa ang kapitalismo at kunsumerismo ay ang pagpuksa sa sistema ng pagpapahalagang kapitalista. Ibig niyang sabihin, sa halip na katigan natin ang pagtitipid, pagtitimpi at pagsisipag, itinuro niya na ang daan patungo sa katapusan ng kapitalismo at konsumerismo ay ang paglulustay, pag-aaksaya at pag-aalay. Isinulat niya: "kung may isang bagay na hindi naisip ni Marx, ito ay ang pagdiskarga, pag-aksaya, pagalay, prodigalidad, laro, at simbolismo."17 Ang simbolikong pagpapalitan ay ang alternatibong landas na inilahad ni Baudrillard na para sa kanya ay tunay na anti-kapitalista.

Medyo mahirap sundan ang landas ng simbolikong pagpapalitan na inilahad ni Baudrillard. Marahil, marami sa atin ang gugustuhin na lamang na labanan ang kapitalismo sa pamamagitan ng pag-iwas sa pamimili ng mga hindi kailangang produkto ng kapitalismo. Ngunit ang ganitong modo ng pakikipaglaban sa kapitalismo at konsumerismo ay magreresulta lamang sa pagdami ng ating ipong pera na sa kalaunan ay hahantong rin sa pagpapalakas lalo ng kapitalismo. Ang ideya ni Baudrillard ay hindi nalalayo sa kaisipan ng Alemang sosyolohista at pilosopong si Max Weber (1864-1920) tungkol sa pagtitipid, pagtitimpi at pagsisipag ng mga sinaunang Protestante, na naging pundasyon sa pagusbong ng kapitalismo. Kung ayon kay Weber naging dahilan ang Protestanteng sistema ng pagpapahalaga sa pag-usbong ng kapitalismo, malakas nga ang puntos ni Baudrillard na mapupuksa lamang ang kapitalismo sa pamamagitan ng pagtakwil sa mga pangunahing elemento

\footnotetext{
${ }^{15}$ See Kellner, "Jean Baudrillard."

${ }_{16}$ Ibid.

${ }^{17}$ Baudrillard, The Mirror of Production, 42.
}

(c) 2015 F.P.A. Demeterio III and Emmanuel C. de Leon http://www.kritike.org/journal/issue 16/demeterio\&de leon june2015.pdf ISSN 1908-7330 
ng sinaunang Protestantismo na nagsisilbi ngayong pundasyunal na sistema ng pagpapahalaga ng kapitalismo. Kaya tunay nga na radikal at makakaliwa ang alternatibong landas na inilahad ni Baudrillard kahit mahirap itong tanggapin bilang kongkretong programa sa pagkilos. Subalit, ang alternatibong landas na ito ay hindi rin nalalayo sa nabubuong hedonistikong landas ng buhay ng ilang indibidwal sa panahon ng postmodernismo.

\section{Postmodernistang Yugto}

Kung sina Marx, Veblen at Saussure ang mga pangunahing impluwensiya ni Baudrillard sa kanyang Marxistang yugto, at sina Mauss at Bataille sa kanyang anti-Marxistang yugto, ang Kanadyanong teorisista ng komunikasyon at pilosopong si Marshall McLuhan (1911-1980) at ang grupong Situationalist ni Lefebvre naman ang kanyang naging inspirasyon sa kanyang postmodernistang yugto na pinasinayaan ng kanyang nabanggit nang aklat na Symbolic Exchange and Death. ${ }^{18}$ Sa 1976 na librong ito nagpangabot ang anti-Marxista at postmodernistang mga yugto ni Baurdrillard.

Alinsunod sa estilo ni McLuhan, gumawa si Baudrillard ng peryodisasyon upang maipakita ang relasyon ng kapanahunan, diskurso ng tanda at orden ng simulacra. Ang unang yugto sa kanyang periodisasyon ay ang kapanahunang midyebal, kung saan ang tanda ay nakatali pa sa kanyang referrent. Kaya sa kapanahunang ito ang tanda ay walang bahid ng kalabuhan. Dahil dito, nagdulot sa lipunan ang midyebal na tanda ng pagkakaroon ng bawat kasapi ng malinaw at hindi nagbabagong katayuan, at matatag na hirarkiya na walang patawad sa sinumang mangangahas sa paggulo sa malinis at malinaw na sistema ng tanda. Paliwanag niya: "Ang mga lipunang may caste, piyudal at makaluma, ay malulupit na lipunan, kung saan ang mga tanda ay limitado sa bilang at balot sa restriksyon. Tangan ng bawat isa sa kanila ang buong interdictory na halaga, at bawat isa ay isang reciprocal na obligasyon sa pagitan ng mga caste, o indibidwal; kaya hindi sila arbitraryo."19 Kaya ang mga indibidwal na sumubok magmunghaki ng panibagong relihiyoso o politikal na interpretasyon at kaayusan ay kadalasang sinusunog o marahas na pinapatay. Para kay Baudrillard, sa kapanahunang midyebal hindi pa umusbong ang orden ng simulacra dahil nakatali pa ang tanda sa kanyang referrent.

Ang pangalawang yugto sa nasabing periodisasyon ay ang kapanahunan ng Renaissance, kung saan nakawala na ang tanda sa kanyang katangi-tanging referrent, at lumutang na ito para maghanap ng panibagong

\footnotetext{
${ }^{18}$ See Kellner, "Jean Baudrillard."

19 Jean Baudrillard, "Symbolic Exchange and Death", in Jean Baudrillard: Selected Writings, ed. by Mark Poster (Stanford, California: Stanford University Press, 1988), 136.

(c) 2015 F.P.A. Demeterio III and Emmanuel C. de Leon http://www.kritike.org/journal/issue 16/demeterio\&de leon june2015.pdf ISSN 1908-7330
}

(cc) BY-NC-ND 
referrent. Kaya sa kapanahunang ito ang tanda ay naging sining. Isinulat niya: "Ang arbitraryong likas ng tanda ay umusbong kapag, sa halip na pagdugtungin ang dalawang indibidwal sa isang hindi nasisirang reciprocity, ang tandang ito ay mag-umpisa, sa signipikasyon, na kumunekta sa disenchanted na uniberso ng mga signified-ang kumon na denominator ng tunay na mundo, na kung saan walang sinuman ang may karagdagan pang obligasyon." 20 Dahil dito, nagdulot sa lipunan ang Renaissance na tanda ng mga bagong kaayusan, posibilidad at alternatibo. Ang sarado at matatag na kaayusang midyebal ay winasak ng Renaissance na tanda. Para kay Baudrillard, sa kapanahunang ito umusbong ang unang orden ng simulacra: ang tanda bilang sining na may taglay na samu't saring kahulugan.

Ang pangatlong yugto sa nasabing periodisasyon ay ang kapanahunan ng rebolusyong industriyal, kung saan ang tanda ay naging produkto na maaaring ipagpalit sa ibang produkto. Kaya sa kapanahunang ito ang tanda ay naging isang kopya mula sa napakaraming kopya na iniluwal ng mga modernong makinarya. Ipinaliwanag niya: "Ito ang mga tandang walang tradisyun ng caste, na hindi naranasan ang mga restriksyon tungkol sa katayuan, at na hindi na kailangang pekehin dahil nilikha sila sa dambuhalang bulto." ${ }^{21}$ Dahil dito, naging abala ang modernong lipunan sa pagreplika ng mga tanda. Para kay Baudrillard, sa kapanahunang ito umusbong and ikalawang orden ng simulacra: ang tanda bilang kopya ng napakaraming kopya. Kung sa unang orden ng simulacra ang tanda ay umiiral bilang teyatro, eskultura, at dibuhung pintura, sa pangalawang orden nito ang tanda ay umiiral bilang sine at litrato.

Ang pang-apat na yugto sa nasabing periodisasyon ay ang kapanahunan ng postmodernismo, kung saan ang tanda ay naging reyalidad na mismo. Sinabi niya: "ang hindi-reyalidad (unreality) ay hindi na namamalagi sa guni-guni o pantasya ... kung hindi sa malahalusinasyong pagkakamukha ng totoo sa kanyang sarili. Para matakasan ang krisis ng representasyon, ang reyalidad ay umikot-ikot sa kanyang sarili sa dalisay na pag-uulit-ulit." 22 Kaya sa kapanahunang ito naging abala ang lipunan sa mga gawain na kung si Marx ang tatanungin ay walang saysay sa usapin ng produksyon: patalastas, midya, impormasyon at komunikasyon. Para kay Baudrillard, sa kapanahunang ito umusbong and ikatlong orden ng simulacra: ang tanda bilang walang referrent at bilang reyalidad na mismo. Naniniwala siya na ang postmodernong lipunan ay labis nang nabighani sa pamang-akit na postmodernong tanda sa puntong kinumpuni na ng nasabing lipunan ang kanyang mga istraktura at proseso paikot sa nasabing tanda.

\footnotetext{
${ }^{20}$ Ibid.

${ }^{21}$ Ibid., 137.

${ }^{22}$ Ibid., 145.
}

(c) 2015 F.P.A. Demeterio III and Emmanuel C. de Leon http://www.kritike.org/journal/issue 16/demeterio\&de leon june2015.pdf

ISSN 1908-7330 
Kung ating babalikan, may apat na yugto ng kasaysayan na binanggit si Baudrillard. Maliban sa midyibal na yugto, ang mga ito ay may kanya-kanyang orden ng simulacra, at kanya-kanyang uri ng tanda. Ang postmodernistang yugto sa kaisipan ni Baudrillard ay nakatuon sa pagaaral sa pangatlong orden ng simulacra, kung saan ang tanda ay lumulutang palayo nang palayo sa kanyang referrent, hindi upang maghanap ng panibagong referrent, tulad ng nangyari sa Renaissance na tanda, kung hindi upang lumago bilang isang independiyenteng tanda na kalaunan ay magiging reyalidad na mismo.

Sa isang panayam ni Jean Nouvel, ipinaliwanag ni Baudrillard ang kulturang namamayani sa panahong postmodernismo. Tinawag niya ang kulturang ito na "aestheticization," na masidhi naman niyang tinutulan. Ipinahayag niya: "tutol ako sa klase ng aestheticization sapagkat palagiang kasama nito ang paglaho: ang paglaho ng bagay, ang paglaho nitong nakatago na posibleng maipakita ng sining at malikhaing obra na kung saan ay lampas pa sa sinasabing aesthetics." ${ }^{23}$ Ang nakatago o sekretong hindi naipakita ng postmodernong kultura ay katulad sa "punctum" na binanggit ni Barthes kaugnay sa potograpiya. Sa pamamagitan ng punctum, naglalaho ang kakayanan nitong maituro ang talagang totoo sa pamamagitan ng mga simbolo. Ang sining sa postmodernong panahon ay hindi na tumuturo sa sekreto. Ang sekretong ito ay hindi lubusang maililipat sa pamamagitan ng isang obra-hinding-hindi makokopya. Ibig sabihin, may pagkakatulad subalit hinding-hindi pa rin matutulad ang reyalidad sa pamamagitan ng isang obra maestra. Ngunit, dahil sa malikhaing obra, nagagawa nitong ibaling ang ating atensyon sa nasabing sekreto o lihim-na-kalaliman. Ito ang nawawala sa postmodernong likhang-sining. Sa pamamagitang ng industriya at teknolohiya ng sining, nababansot ang malikhaing pag-iisip ng tumitingin. Sa pamamagitan ng aestheticization, ang mismong obra na lamang ang lubusang nagpapakita. Naglalaho ang kakayanan nitong ipakita ang sekreto na dapat nitong ipinakikita. Kung kaya nga, hindi nagagawa ng postmodernong sining ang kanyang primordiyal na layunin na akayin ang mga tumitingin sa mga sekretong katotohanan.

May pagkamasalimuot ang pahayag ni Baudrillard na ang postmodernong tanda habang lumalawig palayo sa kanyang referrent ay nagiging reyalidad mismo. Paano nga ba magiging reyalidad ang isang tanda kung alam natin na ito ay nananatili pa ring isang tanda? May pagkametaporikal at may pagka-eksaherado ang takbo ng isip ni Baudrillard sa puntong ito. Ang ibig niyang sabihin ay kadalasan tayong mga tao sa kasalukuyang panahon ay nawalan na ng direktang koneksyon

${ }^{23}$ Jean Baudrillard and Jean Nouvel, The Singular Objects of Architecture (Minneapolis: University of Minnesota Press, 2002), 19.

(c) 2015 F.P.A. Demeterio III and Emmanuel C. de Leon http://www.kritike.org/journal/issue 16/demeterio\&de leon june2015.pdf ISSN 1908-7330

(c) BY-NC-ND 
sa ating mundo at lipunan, dahil ang ating kaalaman sa mga bagay na ito ay ipinamumudmud na sa atin ng telebisyon, radyo, internet at samut-saring babasin. "Samakatuwid," sabi niya, "ang midya ay hindi nagbibigay ng pagkakataon para sa sosyalisasyon, sa halip ay ang kabaligtaran ang ibinibigay nito - ang pamumudmud ng impormasyon sa masa." ${ }^{24}$ Ang mga midyang ito ay hindi naghahandog sa atin ng makatotohan at neyutral na imahen ng mundo. Kadalasan, ang kanilang isinusubo sa atin ay mga imahen na dumaan na sa maraming editing, enhancement, cropping, recycling, at kung anu-ano pa. Sa bandang huli, mahirap nang tukuyin kung ano ba talaga ang kanilang tunay na pinagmulang referrent.

Kadalasan ang mga maningning na espektakulo ng postmodernismo ay binubuo ng mga tandang walang tunay na referrent sa reyalidad, kaya sa puntong ito masasabi nating sila na mismo ang reyalidad, o sa kilalang kataga ni McLuhan ang midyum na ngayon ang mensahe. ${ }^{25}$ Kagaya kunwari ng isang litrato ng isang babaeng nakaswimwear na nasa pahina ng isang fashion magazine, na isang halimbawa ng postmodernong espektakulo na talaga namang kabigha-bighaning pagmasdan. Ngunit ang proseso sa pagbuo ng ganito ka ningning na litrato ay mahaba at kumplikado. Maari itong mag-umpisa sa pag-utos sa isang modelong magpapapayat muna ng ilang libra, sa pagpunas ng langis sa kanyang katawan, sa pag-spray-tan sa kanyang balat, sa pagmanipula sa mga ilaw at kamera sa loob ng estudyo, at sa pag-edit sa inisyal na kuha gamit ang isang software. Sa bandang huli, wala naman talagang ganoon ka ganda at kapayat na babae na nagsilbing referrent ng espektakulong nakaimprinta sa pahina ng naturang fashion magazine. "Nawawala na ang pagtatanghal sa mismong produkto," binigyang diin ni Baudrillard, "nariyan lamang ang malaswa at hungkag na porma. At ang mismong industriya ng patalastas ang ilustrasyon ng walang-katas at hungkag na porma." 26

Ang litrato ng babaeng ito ay isa lamang sa bilyon-bilyong mga espektakulo na lumalaganap sa kasalukuyang panahon. At ang tawag ni Baudrillard dito ay "radical semiurgy," o ang pagdami ng mga tandang walang referrent at mga tandang sila mismo ang reyalidad. Hindi lamang naging pamalit ng reyalidad ang postmodernong tanda; sa patuloy nitong pagiging independiyente sa reyalidad, ito ay naging hyper-reyalidad pa. Ibig sabihin ang postmodernong tanda ay tinuturing nang mas makakatotohan pa kaysa totoo mismo, at mas may reyalidad pa kaysa reyalidad mismo.

\footnotetext{
${ }^{24}$ Jean Baudrillard, Simulacra and Simulation (Ann Arbor, Michigan: University of Michigan Press, 1994), 81.

${ }^{25}$ Ibid., 82.

${ }^{26}$ Ibid., 93.
}

(C) 2015 F.P.A. Demeterio III and Emmanuel C. de Leon http://www.kritike.org/journal/issue 16/demeterio\&de leon june2015.pdf

ISSN 1908-7330 
Kung babalikan natin ang ating nabanggit nang litrato ng babaeng naka-swimwear, ang espektakulong ito ay magiging hyper-reyalidad kung gagamitin ito ng ilang tao na basehan upang husgahan ang mga tunay na babaeng naka-swimwear din. Dahil prinoseso nang mahaba ang litratong nasa fashion magazine, dehadong-dehado at walang kalaban-lang ang mga tunay na babae. Ang sitwasyon ng hyper-reyalidad ay nangyayari kapag ang mga tunay na ang dapat gagaya sa mga prinosesong imahen, sa halip na ang mga imahen ang gagaya sa mga tunay. Kapag ini-isip na natin na dapat ang mga suot nating damit natin ay kahalintulad sa mga damit suot ng mga modelong nasa fashion magazine, na dapat ang ayos ng bahay natin ay kapareho sa mga bahay na nasa architectural manual, na dapat ang pagkain natin ay dapat kamukha sa mga pagkaing nasa cookbook, o na dapat ang kapaligiran natin ay organisado at kasinglinis at kasinglamig sa isang mall o theme park, unti-unti na tayong lumulubog sa kumunoy ng hyperreyalismo.

Ayon kay Baudrillard, ang hyper-reyalismo ay ang pagbubura sa pagkaka-iba ng reyalidad at ng tanda, at tinatawag niya itong implosyon ng pagkaka-iba. Habang patuloy na lumalangoy ang mga tao sa mundo ng hyper-reyalismo, unti-unting nabubura rin ang pagkaka-iba-iba ng kanilang mga konsepto. Dahil sa pagbaha ng kahulugan, na tila isinusuka ng radical semiurgy, nawawalan na ng kahulugan ang mga konsepto. Sa labis-labis na pagtatanghal at pagtatalakay sa tao, lipunan, panlipunang uri, pulitika, liberasyon, rebolusyon, ang mga ito ay nawawalan na rin ng kahulugan. Hanggang ang postmodernong tao ay nakatunganga na lamang sa harap ng mga maniningning na espektakulo ng postmodernong tanda. Sa implosadong mundo ng postmodernismo ang tao ay kadalasang sumisilong na lamang sa anino ng hyper-reyalismo upang pansamantalang malimutan ang sindak na dala ng schizophrenia, at ng pagkalulong natin sa mga bagaybagay, at ng pagtampisaw natin sa mundong nawalan na ng pagkaka-ibaiba.

Kahit ganito kalagim ang nakikita ni Baudrillard, hindi niya hinusgahan ang postmodernong mundo. Marahil alam niya na burado na rin kasi ang pagkaka-iba sa tama at mali. Hindi na rin siya nag-alay pa ng alternatibong programang maaring magligtas uli sa tao mula sa lusak ng implosadong mundo. Marahil alam niya na burado na rin kasi ang pagkakaiba sa mabuti at masama.

\section{Mahahalagang Puntos sa Pilosopiya ni Baudrillard para sa mga Lokal na Pag-aaral ng Teksto at Kultura ng Pilipinas}

Binanggit sa introduksiyon na isa sa mga hangarin ng papel na ito ay ang pagtukoy sa mga aspekto at punto mula sa kaisipan ni Baudrillard

(C) 2015 F.P.A. Demeterio III and Emmanuel C. de Leon http://www.kritike.org/journal/issue 16/demeterio\&de leon june2015.pdf ISSN 1908-7330

(c) $)$ BY-NC-ND 
na may maiaambag para sa lalong pagpapayaman sa teoretikal na korpus ng lokal nating araling kultural at araling Pilipino. Kaya sa seksiyong ito hahalawin natin mula sa kanyang pilosopiya ang ilang aspekto at punto na magagamit natin sa pagpapayabong sa ating sariling tekstuwal at kultural na mga pagsusuri.

Una, malaki ang potensyal ng ginawang pag-aaral ni Baudrillard sa kanluraning penomenon ng pagkunsumo ng tanda sa pag-unawa kung gaano kalaganap ang kaparehong penomenon dito sa ating bansa. Gamit ang nasabing pag-aaral, maaari din nating alamin kung may pagkakaiba ba ang kanluraning pagkunsumo ng tanda sa ating sariling pagkunsumo ng tanda. Gayong kapansin-pansin ang angas, yabang at pagiging partikular nating mga Pilipino sa ating panlipunang katayuan, mas matindi kaya ang ating pagkunsumo ng tanda? Sinu-sino ang nagpapalaganap ng mga tandang ito at paano nila ito pinapalaganap? Maaari din nating dalhin pa si Baudrillard sa ating pagsisid sa medyo kakaiba nating pagkunsumo ng mga peke at pinekeng produkto. Maiuugnay kaya ang gawain kaugaliang ito sa konsepto ng pagkunsumo ng tanda ni Baudrillard?

Pangalawa, interesante ang konsepto ni Baudrillard tungkol sa mamahalin at prestihiyosong mga produkto at kung paano ang mga ito itinanghal ng mga tusong kapitalista. Maaaring gamitin ang konseptong ito bilang lente ng pagsusuri sa sarili nating mga produkto na itinuturing nating prestihiyoso. Ang mga imported na bagay na halos sinasamba na ng mga Pilipino ay kahalintulad ba sa mga mamahalin at prestihiyosong produkto na ayon kay Baudrillard ay gawa ng monopolyong kapitalismo, o kahalintulad lamang ang mga ito sa mga eksotikong produkto ng mas lumang kapitalismo? May mga lokal ba tayong produkto na itinatanghal din ng mga Pilipinong kapitalista bilang mga mahahalin at prestihiyosong bagay? Kung sakali meron man, may pagkakaiba ba ang pagtanghal ng Pilipinong kapitalista sa kanilang mamahalin at prestihiyosong produkto sa pagtanghal ng mga kanluraning kapitalista sa kanilang kaparehong produkto? Kung sakali meron man tayong mga lokal na mamahalin at prestihiyosong mga bagay, paano kaya ang mga ito lumalaban sa mga imported na bagay, prestihiyoso man o hindi, na pinapahalagahan nang husto ng mga Pilipino? May potensyal ang konsepto ni Baudrillard hindi lamang sa postkolonyal na pagsusuri ng kunsumerismong Pilipino kung hindi pati na rin sa postkolonyal na pakikibaka laban sa ating neo-kolonyal na kalagayan. Ito ay dahil ang ibinunyag niyang gawain ng mga tusong kanluraning kapitalista na may kinalaman sa artipisyal na konstruksyon ng prestihiyo ng kanilang produkto ay posibleng gayahin ng mga nasyunalistang kapitalistang Pilipino. Kung meron man tayong ganitong uri ng mga kapitalistang Pilipino, artipisyal ding buuin ang prestihiyo ng 
produktong Pilipino sa antas na magiging mas prestihiyoso pa ang mga ito kaysa katumbas nilang imported na produkto.

Pangatlo, mahalaga ang ginawang konseptuwal na pagkakaiba ni Baudrillard sa pagitan ng monopolyong kapitalismo at kapitalismo ng kompetitibong merkado para mabigyang diin na ang katotohanang ang kapitalismo ay hindi isang monolitiko at hindi nagbabagong kumpigurasyon, kung hindi isang sistema na umiinog sa paglipas ng panahon at posibleng nag-iibang anyo kumporme sa kanyang konteksto. Ang aral na ito ay maaaring gamitin para suriin nating mabuti kung ano na ba ang kasalukuyang anyo ng kapitalismong umiiral sa bansa natin, o hindi kaya kung ano ba talaga ang kasalukuyang modo ng produksyong na umiiral sa bansa natin. Pero dapat nating asahan na ang mga lokal na anyong ito ay higit pang mas kumplikado kaysa modelong binuo ni Baudrillard para sa kanluran, dulot ng katotohanang ang Pilipinas ay isang neo-kolonyal na estado at isang maliit at mahinang manlalaro sa larangan ng globalisasyon.

Pang-apat, ang pagbatikos ni Baudrillard sa Marxismo ay maaari nating gawing modelo sa pagsuri sa uri, o mga uri, ng Marxismong dinidiskurso at itinataguyod dito sa ating bansa. Ano nga ba ang hugis ng utopia, o mga utopia, na ipinapangako ng mga ideolohiya at kilusang ito? Kaya nga bang labanan ng mga nasabing ideolohiya at kilusan ang kasalukuyang anyo ng kapitalismo, o ng anumang modo ng produksyon, na umiiral sa ating bansa? Makabuluhan pa bang pag-usapan ang pilosopiya ni Marx sa konteksto ng kasalukuyan nating lipunan? Maaari din nating pagnilayan ang kapangyarihan at praktikalidad ng iminumungkahi ni Baudrillard na heneral na ekonomiya at simbolikong pagpapalitan bilang landas patungo sa wakas ng kapitalismo. Sa konteksto natin bilang isang umuunlad pa lamang na bansa, ang heneral na ekonomiya at simbolikong pagpapalitan ba ay mga angkop at makatarungang estratihiya para labanan ang kapitalismo? Anu-ano kaya ang mga mas tugmang estratihiya na makapangyarihan, praktikal at reyalistiko nating magamit para puksain ang mga masamang elemento at aspekto ng kapitalismo at globalisasyon?

Panglima, iteresante ang konsepto ng hyperreyalismo ni Baudrillard na gamiting lente ng pagsusuri kung umiiral din ba ito, kung gaano kalaganap ito, at kung ano ang anyo nito sa ating bansa. Sa isang banda, katanggap-tanggap isipin na mahina ang presensya ng hyperreyalismo sa Pilipinas dulot ng katotohanang ito ay nililikha ng makabagong teknolohiya at sa konteksto ng isang bansang malawak ang technological divide maliit lamang ang tsansa nitong saklawan ang buong populasyon. Ngunit sa kabilang banda, katanggap-tanggap ding isipin na malakas ang presensya ng hyperreyalismo sa Pilipinas dulot ng katotohanang sa mga rehiyon na matatagpuan ang mga makabagong

(C) 2015 F.P.A. Demeterio III and Emmanuel C. de Leon http://www.kritike.org/journal/issue 16/demeterio\&de leon june2015.pdf ISSN 1908-7330

(c) BY-NC-ND 
teknolohiyang ito, katulad ng mga metropolitan area, talaga namang babad na babad ang nagkukumpulang mga Pilipino sa mga terminal ng hyperreyalismo, katulad ng telebisyon, sine, mall, computer, radyo, mga babasahin, at mga kahalintulad na bagay/sistema. Ano kaya ang epekto ng hyperreyalismo sa kamalayan ng mga Pilipinong binighani na nito? May pagkakaiba kaya ang kamalayan ng Pilipinong hindi pa lumubog at ng mga Pilipinong lumubog na sa kumunoy ng hyperreyalismo?

Department of Filipino, De La Salle University-Manila, Philippines Department of Philosophy, University of Santo Tomas, Philippines

\section{References}

Baudrillard, Jean and Nouvel, Jean, The Singular Objects of Architecture (Minneapolis: University of Minnesota Press, 2002).

Baudrillard, Jean, "Symbolic Exchange and Death," Jean Baudrillard: Selected Writings (Stanford, California: Stanford University Press, 1988).

"The System of Objects," in Jean Baudrillard: Selected Writings (Stanford, California: Stanford University Press, 1988). Simulacra and Simulation (Ann Arbor, Michigan: University of Michigan Press, 1994). The Mirror of Production (St. Louis, Missouri: Telos Press, 1975).

Gane, Mike ed., Baudrillard Live: Selected Interviews (London: Routledge, 1993).

Genosko, Gary, McLuhan and Baudrillard: the Masters of Implosion (London: Routledge, 1999).

Hefner, Robert, "Baudrillard's Noble Anthropology: The Image of Symbolic Exchange in Political Economy," in SubStance, 6/7:17 (Autumn 1977).

Horrocks, Chris, Introducing Baudrillard (New York: Totem Books, 1996).

Kellner, Douglas, "Jean Baudrillard," in The Stanford Encyclopedia of Philosophy, (2014), <http://plato.stanford.edu/archives/win2014/ entries/baudrillard/>, 30 March 2015.

Lane, Richard, Jean Baudrillard (London: Routledge, 2000).

Merrin, William, Baudrillard and the Media: a Critical Introduction (Cambridge: Polity Press, 2005).

Poster, Mark, "Introduction," in Jean Baudrillard: Selected Writings (Stanford, California: Stanford University Press, 1988).

Sarup, Madan, An Introductory Guide to Post-Structuralism and Postmodernism (Athens: University of Georgia Press, 1993). 